\title{
Complex Impedance as a Diagnostic Tool for Characterizing Thermal Detectors
}

\author{
John E. Vaillancourt ${ }^{\mathrm{a}}$ \\ Physics Department, University of Wisconsin, 1150 University Ave., Madison, WI 53706
}

\begin{abstract}
The complex ac impedance of a bolometer or microcalorimeter detector is easily measured and can be used to determine thermal time constants, thermal resistances, heat capacities, and sensitivities. Accurately extracting this information requires an understanding of the electrical and thermal properties of both the detector and the measurement system. We show that this is a practical method for measuring parameters in detectors with moderately complex thermal systems.
\end{abstract}

PACS numbers: 07.20.Fw; 07.57.Kp; 84.37.+q; 85.25.Am; 85.30.De

Keywords: bolometers; microcalorimeters; impedance

\section{INTRODUCTION}

Thermal detectors are used in a number of fields ranging from particle and plasma physics to astrophysics. The two commonly used forms of these detectors are bolometers to measure incident power and microcalorimeters to measure total energy. Despite these different applications their construction and operation principles are similar. In general, they are composed of an absorbing element to collect incident radiation or particles, a resistive thermometer coupled to the absorber, and a weak thermal link connecting the thermometer to a heat sink.

The simple model treated in most analytical descriptions consists of a lumped heat capacity connected to a heat sink through a weak thermal link [Fig. 1(a)]. The theory predicting the responsivity, noise properties, and energy resolution of these detectors has been thoroughly developed. ${ }^{1-4}$ However, more complex thermal models are often needed to describe real detectors. The internal construction of these detectors often introduces additional time constants and noise terms which can have major detrimental effects on performance. ${ }^{5-8}$

A detector's performance can be predicted and optimized for specific applications $s^{4,8-10}$ if the thermal and electrical properties of their components are known. Thermal conductivities, heat capacities, and sensitivities can be extracted from measurements of the resistance vs. temperature relation, dc $I V$ (current-voltage) curves, and time constants. ${ }^{1,3,11,12}$ The thermal time constant can be found by measuring either the detector's impulse response function in the time domain ${ }^{5,11,13,14}$ or its complex ac impedance $Z(\omega) \equiv \mathrm{d} V / \mathrm{d} I$ in the frequency domain. ${ }^{2,3}$ In this paper we discuss the impedance method, which can often be used to find more precise parameter values than the impulse response method. ${ }^{15}$

We will show that the internal heat capacities and thermal conductivities of both simple and complex detectors, along with the thermometer temperature sensitivity and thermometer voltage (or current) sensitivity can be determined from measurements of dc $I V$-curves and the ac impedance. As examples of this technique we fit impedance data to the ideal detector model and one of the more complex thermal circuits presented by Galeazzi and McCammon ${ }^{6}$. This complex model in-

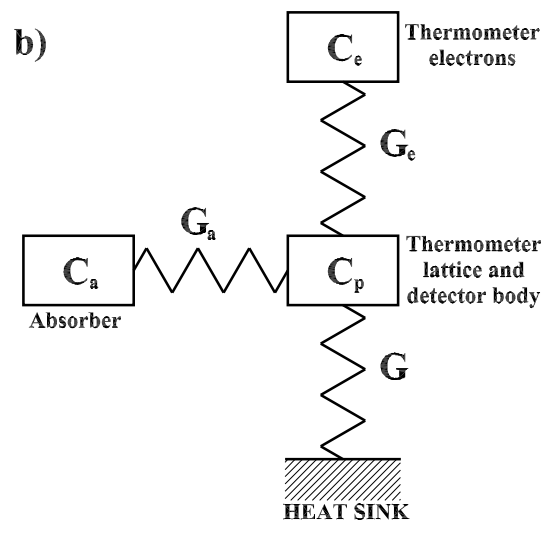

FIG. 1: a) The ideal model of a thermal detector consists of a lumped heat capacity $C$ connectecd to a heat sink through a weak thermal link $G$. b) A more realistic model that includes internal couplings. ${ }^{6}$

cludes electron-phonon decoupling ${ }^{16-18}$ in the thermometer and a thermal resistance between the absorber and thermometer.

We begin with a review of the impedance of the simplest detector model in Sec. II. Section III presents example fits of simple and complex thermal models to impedance data. In Sec. IV we discuss the effects of stray electrical capacitances and inductances in the bias and readout circuits. While the examples presented here utilize a voltage readout circuit, the equations can readily be transformed to current readout.

\section{THE SIMPLE THERMAL DETECTOR}

The simplest, or ideal, detector consists of a single lumped heat capacity $C$ connected to a heat sink through a weak thermal link of conductivity $G$ [Fig. 1(a)]. This model has been widely discussed by other authors; ${ }^{1-3,6}$ we briefly review their results here.

The dynamic impedance for this simple model is given by

$$
Z(\omega)=\frac{R}{\left(1-\beta_{v}\right)} \frac{1+L_{v}+\mathrm{j} \omega \tau}{1-L_{v} /\left(1-\beta_{v}\right)+\mathrm{j} \omega \tau}
$$


where

$$
\begin{aligned}
L_{v} & =\text { dimensionless gain } \equiv \frac{\alpha_{v} P}{G T} \\
R & =\text { detector resistance } \\
T & =\text { detector temperature } \\
P & =\text { Joule power dissipated in detector }=V^{2} / R \\
G & =\text { thermal conductivity to heat bath }=\mathrm{d} P / \mathrm{d} T \\
\tau & =\text { thermal time constant }=C / G \\
\omega & =\text { angular frequency } \\
\alpha_{v} & =\text { thermometer sensitivity at constant voltage } \\
& =\left.\frac{T}{R} \frac{\partial R}{\partial T}\right|_{V} \\
\beta_{v} & =\text { thermometer voltage dependence at constant } T \\
& =\left.\frac{V}{R} \frac{\partial R}{\partial V}\right|_{T}
\end{aligned}
$$

For a linear thermometer the resistance is dependent only on its temperature and $\beta_{v}=0$. However, this is not generally the case so we will retain the term here.

Equation (1) can be rewritten as

$$
Z(\omega)=\frac{Z_{0}+Z_{\infty}}{2}+\frac{Z_{0}-Z_{\infty}}{2} \frac{1-\mathrm{j} \omega \bar{\tau}_{z}}{1+\mathrm{j} \omega \bar{\tau}_{z}}
$$

where

$$
\begin{aligned}
Z_{\infty} & \equiv \lim _{\omega \rightarrow \infty} Z(\omega)=R /\left(1-\beta_{v}\right) \\
Z_{0} & \equiv Z(0)=Z_{\infty} \frac{1+L_{v}}{1-L_{v} /\left(1-\beta_{v}\right)}, \text { and } \\
\bar{\tau}_{z} & \equiv \frac{Z_{0}+R}{Z_{\infty}+R} \tau .
\end{aligned}
$$

Equation (8) describes a semi-circle in the complex plane of radius $\frac{1}{2}\left|Z_{\infty}-Z_{0}\right|$ centered on the real axis at $\frac{1}{2}\left(Z_{\infty}+\right.$ $\left.Z_{0}\right)$. The frequency at the peak of the circle is given by the dynamic time constant $\bar{\tau}_{z}$. This time constant is not the same as the effective time constant, which describes the effect of electro-thermal feedback on the detector response and is dependent on the relative resistance values of the detector and load resistor. The dynamic time constant is a property of the detector only. Equations (8)-(11) are equivalent to those given by Mather ${ }^{3}$.

When $\alpha_{v}>0$ [as is the case for a superconducting transition-edge sensor (TES)] it is possible for the impedance to become infinite $\left[L_{v}=1-\beta_{v}\right.$ in Eq. (10)]. To avoid this, one might instead measure the complex admittance $A(\omega)=1 / Z(\omega)$. The relations describing the admittance are easily found by transforming the preceding relations using the dual circuit theorem, namely

$$
\begin{aligned}
I & \leftrightarrow V, \\
R & \leftrightarrow S(\equiv 1 / R), \\
Z & \leftrightarrow A,
\end{aligned}
$$

$C$ (parallel capacitance) $\leftrightarrow L$ (series inductance 15 )
Since it is customary to keep $\alpha$ and $\beta$ as derivatives of resistance rather than conductance, the signs of these quantities will change:

$$
\begin{gathered}
\left.\frac{T}{S} \frac{\partial S}{\partial T}\right|_{I}=-\left.\frac{T}{R} \frac{\partial R}{\partial T}\right|_{I} \equiv-\alpha_{i}, \\
\left.\frac{I}{S} \frac{\partial S}{\partial I}\right|_{T}=-\left.\frac{I}{R} \frac{\partial R}{\partial I}\right|_{T} \equiv-\beta_{i}, \\
L_{i}=\frac{\alpha_{i} P}{G T} .
\end{gathered}
$$

The complex admittance is then

$$
A(\omega)=\frac{A_{0}+A_{\infty}}{2}+\frac{A_{0}-A_{\infty}}{2} \frac{1-\mathrm{j} \omega \bar{\tau}_{a}}{1+\mathrm{j} \omega \bar{\tau}_{a}}
$$

where

$$
\begin{aligned}
A_{\infty} & =S /\left(1+\beta_{i}\right), \\
A_{0} & =A_{\infty} \frac{1-L_{i}}{1+L_{i} /\left(1+\beta_{i}\right)}, \text { and } \\
\bar{\tau}_{a} & =\frac{A_{0}+S}{A_{\infty}+S} \tau .
\end{aligned}
$$

With these equations we see that the path a negative detector $\left(\alpha_{v}<0\right)$ traces through the complex impedance plane is equivalent to the path traced through the complex admittance plane for a positive detector $\left(\alpha_{i}>0\right)$.

\section{IMPEDANCE MEASUREMENTS}

Figure 2 shows examples of impedance measurements and fitted models for three different microcalorimeter detectors. The first example is for a doped silicon thermistor with no absorber [Figs. 2(a) and 2(b)]. The single semi-circle is adequately represented by Eq. (8) with the parameters shown in Table I. The mismatch between the data and models at high frequency is most likely due to incomplete modeling of stray capacitances (see Sec. IV). In the other two examples, composite absorbers have been glued to the detectors [Figs. 2(c)-2(f)]. The more complex behavior of $Z(\omega)$ indicates significant thermal resistances which can be reasonably fit by the thermal model of Fig. 1(b). The complex impedance for this model is derived by Galeazzi and McCammon ${ }^{6}$ as their Eq. (110) (note that they use $\beta_{i}$ rather than $\beta_{v}$ ).

The complete characterization of these thermal detectors includes the sensitivities of the thermistor $\alpha$ and $\beta$, and the heat capacities and thermal conductivities of all constituent parts. However, these parameters cannot be determined from measurements of the impedance alone. The impedance of the ideal thermal model is completely described by the three parameters $Z_{0}, Z_{\infty}$, and $\bar{\tau}_{z}$ in Eq. (8), which are determined by fitting the measured $Z(\omega)$. 

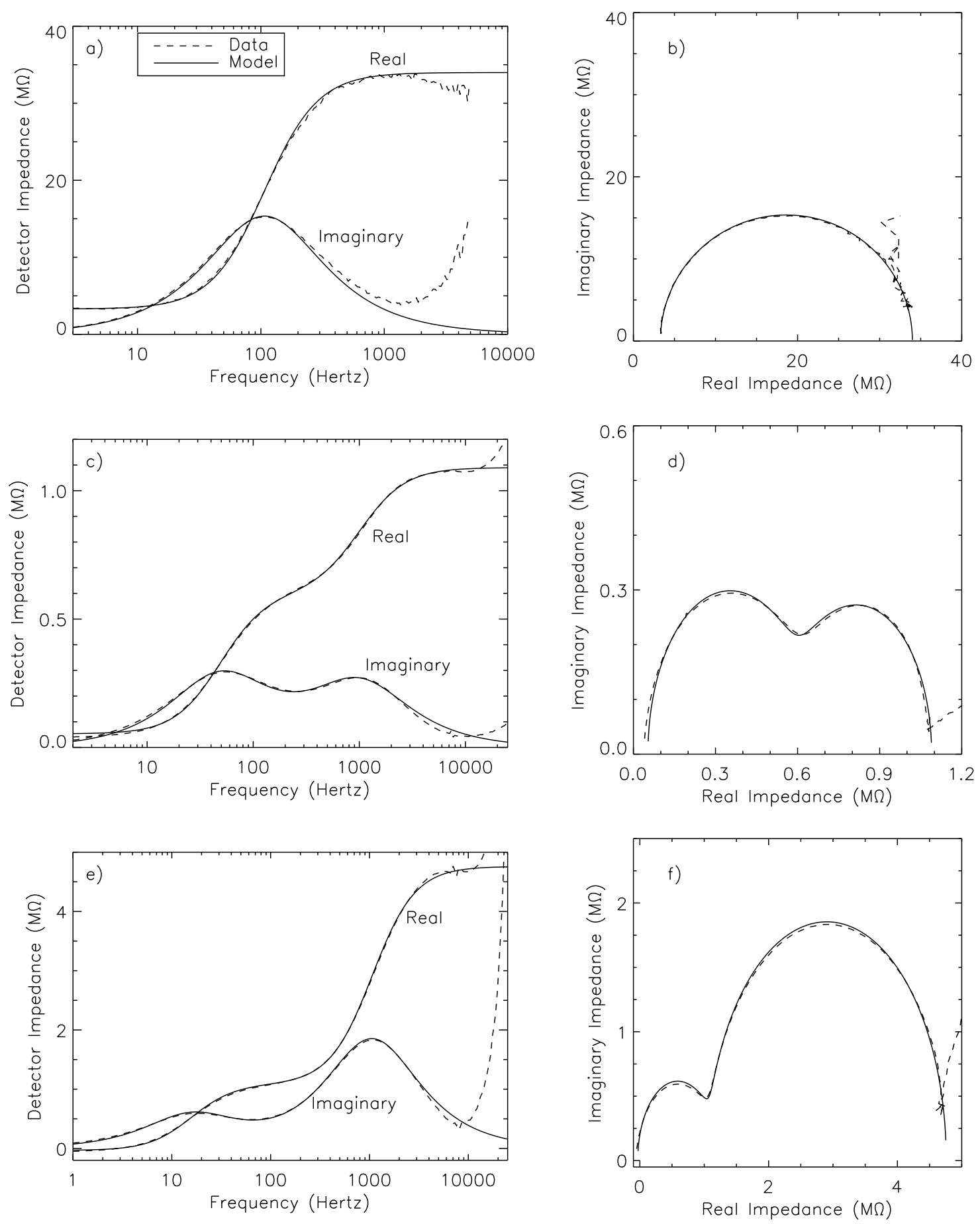

FIG. 2: Examples of impedance measurements for a bare thermometer [(a) and (b)] and thermometers with absorbers [(c)-(f)]. Data are shown as dotted lines and the fitted model as solid lines. The figures on the left $[(\mathrm{a}),(\mathrm{c})$, and (e)] show the frequency variation of the real and imaginary parts of the detectors' complex impedance while the figures on the right [(b), (d), and (f)] plot the same data in the complex plane. The divergence of the fits at high frequency is due to limitations of modeling the electrical circuit stray capacitances. The x:y axis ratio is 1:1 in (b), and 2:1 in (d) and (f). For clarity, data above $5 \mathrm{kHz}$ have been removed in (a) and (b). The fit model parameters for (a)-(d) are given in Table I. 
TABLE I: Example detector parameters

\begin{tabular}{lccl}
\hline \hline Parameter & Bare $^{a}$ & Absorber $^{b}$ & Method $^{c}$ \\
\hline$R(\mathrm{M} \Omega)$ & 36 & 1.3 & dc measurement \\
$P(\mathrm{pW})$ & 0.22 & 7.0 & dc measurement \\
$T(\mathrm{mK})$ & 70 & 140 & $R$-vs- $T$ calibration \\
$\alpha_{v}$ & -7.5 & -4.5 & $R$-vs- $T$ calibration \\
$G(\mathrm{pW} / \mathrm{K})$ & 35 & $230^{d}$ & dc IV-curve \\
$C_{\mathrm{p}}(\mathrm{pJ} / \mathrm{K})$ & $\ldots$ & 0 & fixed \\
$\bar{\tau}_{z}(\mathrm{~ms})$ & 1.5 & $\ldots$ & Impedance fit \\
$Z_{0}(\mathrm{M} \Omega)$ & 3.3 & 0.053 & Impedance fit \\
$Z_{\infty}(\mathrm{M} \Omega)$ & 34 & 1.1 & Impedance fit \\
$\beta_{v}$ & -0.058 & -0.17 & Eq. (9) \\
$L_{v}$ & -0.83 & -0.91 & Eq. (10) \\
$\tau(\mathrm{ms})$ & 2.7 & $\ldots$ & Eq. (11) \\
$\alpha_{v}$ & -9.0 & -4.2 & Eq. $(2)$ \\
$C_{\mathrm{e}}(\mathrm{pJ} / \mathrm{K})$ & 0.093 & 0.13 & Eq. (5), Impedance fit \\
$C_{\mathrm{a}}(\mathrm{pJ} / \mathrm{K})$ & $\ldots$ & 0.64 & Impedance fit \\
$G_{\mathrm{a}}(\mathrm{pW} / \mathrm{K})$ & $\ldots$ & 460 & Impedance fit \\
\hline \hline
\end{tabular}

${ }^{a}$ Bare thermistor shown in Figs. 2(a)-(b)

${ }^{b}$ Thermistor with absorber shown in Figs. 2(c)-(d)

${ }^{c}$ Method used to measure the indicated parameter

${ }^{d}$ Series combination of $G$ and $G_{\mathrm{e}}$

${ }^{e} C_{\mathrm{e}}$ for the bare thermistor is found from Eq. (5); it is fit directly for the thermistor with absorber.

A dc determination of the resistance yields $\beta_{v}, L_{v}$, and $\tau$ through Eqs. (9)-(11). However, $\alpha_{v}, C$, and $G$ cannot be separated from $L_{v}$ and $\tau$ without another independent measurement. For this last measurement, $G(T)$ is fitted to dc $I V$-curves.

The data in Figs. 2(c)-2(f) were fit using the model in Fig. 1(b), which separates the detector heat capacity into contributions from the absorber $C_{\mathrm{a}}$, thermistor electrons $C_{\mathrm{e}}$, and thermistor phonons $C_{\mathrm{p}}$, connected by the labeled thermal conductivities. A complete characterization requires eight parameters: three heat capacities, three thermal conductivities, and two thermometer sensitivities. In principle, this model contains three separate thermal time constants which could appear as three separate circles in the complex impedance data. In practice, the phonon heat capacity in our doped silicon thermistors is much smaller than either the electron or absorber heat capacities, placing this third time constant at frequencies beyond the practical measurement range. Therefore, $C_{\mathrm{p}} \approx 0$ and $G$ and $G_{\mathrm{e}}$ can be replaced by their series combination. The remaining six parameters are still perfectly correlated in fits to the impedance alone so independent measurements are required to separate them, just as in the ideal model.

The procedures outlined above for characterizing thermal detectors contain at least one known systematic error. The fits of $G(T)$ to $I V$-curves assume that $\beta_{v}=0$, while the impedance fits and resistance measurements clearly indicate that $\beta_{v} \neq 0$ (see Table I). This prob- lem could be alleviated using an iterative procedure (refitting the $I V$-curves with the non-zero $\beta_{v}$ ) or by performing simultaneous fits to both the $I V$-curves and impedance. Without these corrections the small values of $\beta_{v}$ measured in the doped silicon thermometers $\left(\left|\beta_{v}\right|<0.2\right)$ introduce only a few percent uncertainty in the $I V$-curve determination of $G(T)$. These required corrections may be larger in TES detectors which can have relatively large values of $\beta_{i}(\approx 1-3) .{ }^{15}$

\section{READOUT CIRCUITS}

\section{A. The Transfer Function}

Figure 3 illustrates two equivalent circuits for biasing thermal detectors, either of which can be used to measure the impedance. The first utilizes voltage readout [Fig. $3(\mathrm{a})$ ], the other current readout [Fig. 3(b)]. Our technique for measuring the dynamic impedance is to add a small ac signal to the dc bias and measure the resulting complex transfer function as a function of frequency. The transfer functions are defined as $T(\omega) \equiv V_{\text {out }}(\omega) / V_{\text {in }}(\omega)$ for voltage readout and $T(\omega) \equiv I_{\text {out }}(\omega) / I_{\text {in }}(\omega)$ for current readout. The ac signal can be either a random noise source with a bandwidth spanning the frequencies of interest or a sinusoidal source that can be scanned through the desired frequency range. We use a commercial spectrum analyzer to measure the complex transfer function using both of these methods. The sine-sweep method could also be implemented with a two-phase lock-in amplifier and swept sine generator.

\section{B. Electrical Strays}

For an ideal bias circuit the transfer function would simply be the voltage or current divider formed by $R_{L}$ and $Z(\omega)$. In practice, additional reactive components exist in the form of stray capacitances and/or inductances. These stray reactances will introduce additional phase and amplitude shifts in $T(\omega)$, but it is possible to correct for these effects if the strays in the bias circuit can be accurately modeled and measured.

The stray reactances can be determined by measuring the transfer function of the circuit with the reactive part of $Z(\omega)$ removed. This is done by either replacing the detector with a pure resistor or by measuring the transfer function of an unbiased $\left(\left\langle V_{\mathrm{in}}\right\rangle=\left\langle I_{\mathrm{in}}\right\rangle=0\right)$ detector. If the unbiased transfer function is measured with a sufficiently small ac signal, such that the detector dissipates negligible power $(P \approx 0)$, then the detector is purely resistive [see Eqs. (1) and (2)]. Any observed reactive component under these conditions must be due to stray reactances in the circuit.

The dominant reactances in our voltage readout circuit for silicon detectors are shunt capacitances across 

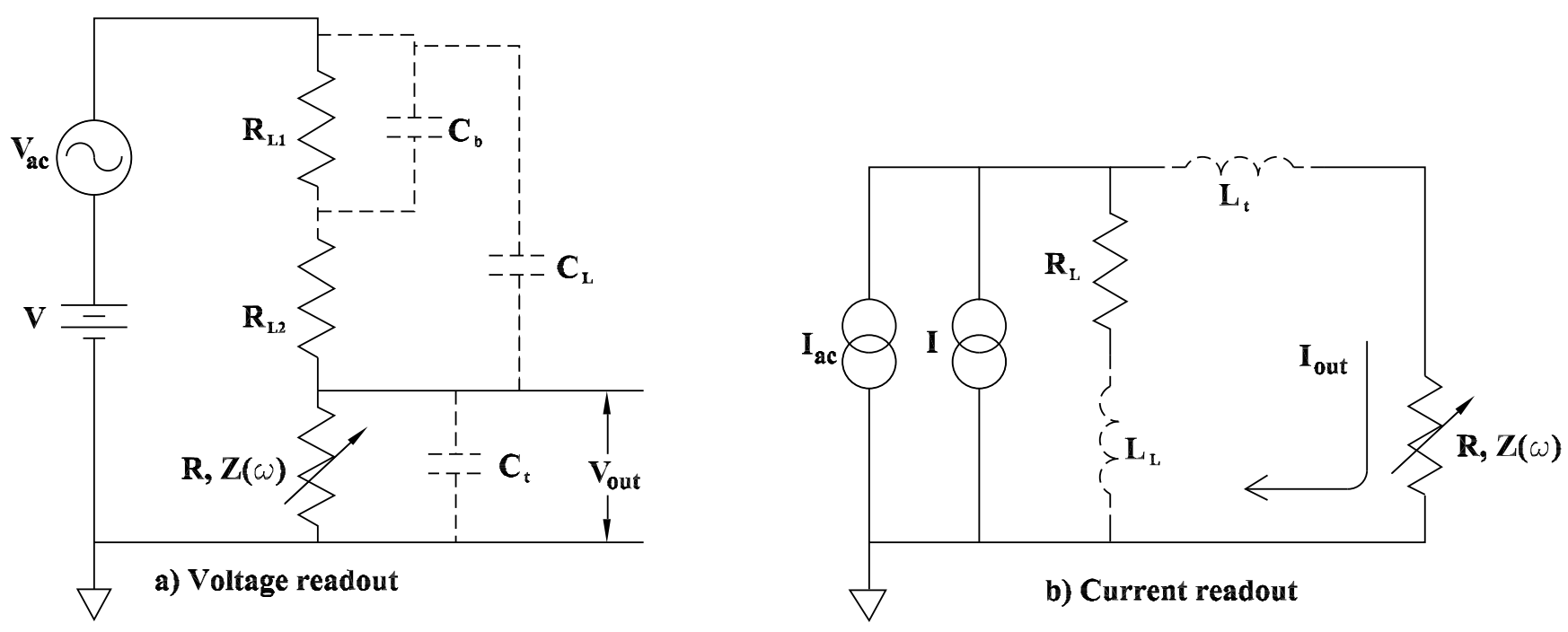

FIG. 3: Bias circuits for measuring detector transfer functions. Both circuits consist of a thermometer impedance $Z(\omega)$, resistance $R$, and load resistor $R_{\mathrm{L}}$. a) Voltage readout circuit for doped silicon thermometers. The capacitors connected by dotted lines are used to model stray capacitances in the system. $C_{\mathrm{L}}$ and $C_{\mathrm{t}}$ are shunt capacitances across the load resistor and thermometer, respectively. The load resistor is one physical resistor, but it has been split in this schematic $\left(R_{\mathrm{L}}=R_{L_{1}}+R_{L_{2}}\right)$ in order to partially model the distributed capacitances $C_{\mathrm{b}}$. b) Current readout circuit for TES. The inductors shown as dotted lines are used to model stray inductances in series with the thermometer, $L_{\mathrm{t}}$, and load resistor, $L_{\mathrm{L}}$. If $C_{\mathrm{b}}=0$ these two circuits are dual equivalents and all equations describing one system can be transformed to the other system using the substitutions in Eqs. (12)-(15).

the detector and load resistor $\left(C_{\mathrm{t}}\right.$ and $C_{\mathrm{L}}$ in Fig. 3, respectively). For this model circuit the transfer function is given by

$$
T(\omega)=\frac{V_{\text {out }}}{V_{\text {in }}}=\frac{Z(\omega)}{R_{\mathrm{L}}+Z(\omega)} \frac{1+\mathrm{j} \omega \tau_{\mathrm{L}}}{1+\mathrm{j} \omega \tau_{\mathrm{t}}}
$$

where

$$
\begin{aligned}
\tau_{\mathrm{L}} & =R_{\mathrm{L}} C_{\mathrm{L}} \quad \text { and } \\
\tau_{\mathrm{t}} & =\frac{C_{\mathrm{L}}+C_{\mathrm{t}}}{R_{\mathrm{L}}^{-1}+Z(\omega)^{-1}} .
\end{aligned}
$$

This simple model for the electrical strays also traces a semi-circle in the complex plane if the detector impedance is completely real, $Z(\omega)=R$.

$$
T(\omega)=\frac{T_{0}+T_{\infty}}{2}+\frac{T_{0}-T_{\infty}}{2} \frac{1-\mathrm{j} \omega \tau_{\mathrm{t}}}{1+\mathrm{j} \omega \tau_{\mathrm{t}}}
$$

where

$$
\begin{aligned}
T_{\infty} & \equiv \lim _{\omega \rightarrow \infty} T(\omega)=\frac{C_{\mathrm{L}}}{C_{\mathrm{L}}+C_{\mathrm{t}}}, \text { and } \\
T_{0} & \equiv T(0)=\frac{R}{R_{\mathrm{L}}+R}
\end{aligned}
$$

The dual circuit theorem can be used to transform these equations from the voltage readout circuit of Fig. 3 (a) to the current readout circuit of Fig. 3(b). Equations
(23)-(25) are then

$$
\begin{aligned}
T(\omega)=\frac{I_{\text {out }}}{I_{\text {in }}} & =\frac{A(\omega)}{S_{\mathrm{L}}+A(\omega)} \frac{1+\mathrm{j} \omega \tau_{\mathrm{L}}}{1+\mathrm{j} \omega \tau_{\mathrm{t}}} \\
& =\frac{R_{\mathrm{L}}}{R_{\mathrm{L}}+Z(\omega)} \frac{1+\mathrm{j} \omega \tau_{\mathrm{L}}}{1+\mathrm{j} \omega \tau_{\mathrm{t}}}
\end{aligned}
$$

where

$$
\begin{aligned}
\tau_{\mathrm{L}} & =L_{\mathrm{L}} / R_{\mathrm{L}} \quad \text { and } \\
\tau_{\mathrm{t}} & =\frac{L_{\mathrm{L}}+L_{\mathrm{t}}}{R_{\mathrm{L}}+Z(\omega)} .
\end{aligned}
$$

Equations (26)-(28) can be similarly transformed.

Real strays are complex, involving distributed reactances rather than (or in conjunction with) the simple parallel capacitances and series inductances discussed here. It is the accuracy of the circuit model, not the values of the circuit elements (both real and stray) that limits the maximum useful frequency for impedance measurements. This will particularly limit the accuracy with which $Z_{\infty}$ and $\beta_{v}$ (or $A_{\infty}$ and $\beta_{i}$ ) can be measured.

No stray inductances have been included in Fig. 3(a) as they are generally negligible for high-impedance silicon detectors. Similarly, the effect of stray capacitances can be neglected for the very low impedance TES detectors. In this case the stray inductances shown in Fig. 3(b) can be significant. However, these stray inductances still seem to be less of a problem for the low-impedance detectors than stray capacitances are for high-impedance detectors. As a result, the impedance of a TES can often 


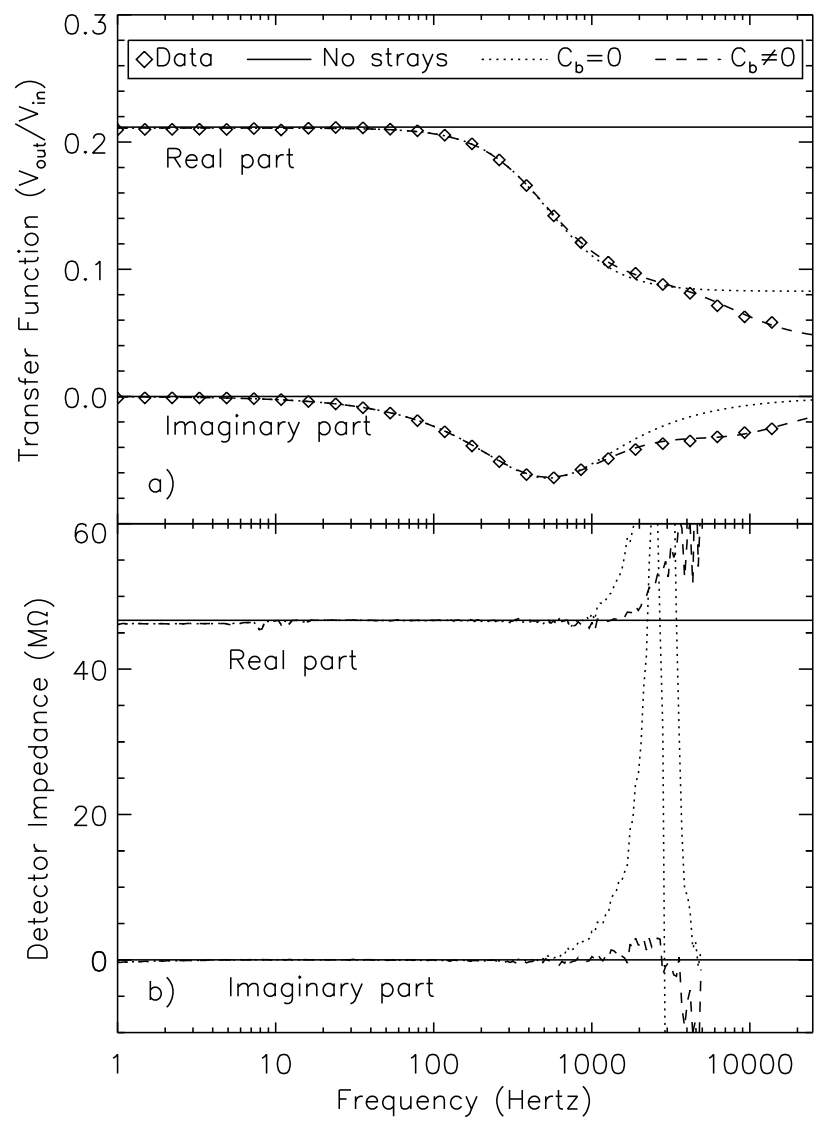

FIG. 4: Example of an unbiased transfer function. a) The measured complex transfer function (diamonds represent every tenth data point) and fits to two stray capacitance models [dotted and dashed lines; Fig. 3(a)]. If no strays were present the data would follow the solid lines. b) The inferred detector impedance after correcting for each stray model (dotted and dashed lines) is compared with an ideal resistor (solid lines). One model uses only shunt capacitances across the detector and load resistor $\left(C_{\mathrm{b}}=0\right)$ while the other also includes a distributed capacitance within the load resistor $\left(C_{\mathrm{b}} \neq 0\right)$. The impedance data above $5 \mathrm{kHz}$ have been removed from the bottom plot for clarity.

be measured up to higher frequencies (tens of $\mathrm{kHz})^{15,19}$ than the silicon detectors (a few $\mathrm{kHz}$ ).

We note that the utility of the impedance method is not limited to only those systems which can be described by the bias circuit models in Figure 3. It is easily extended to any bias circuit whose frequency dependent transfer function can be modeled and measured. This includes bridge circuits and long transmission lines, such as those often employed in fusion research. ${ }^{5,11,12,14}$

\section{Measuring Stray Capacitances}

The shunt capacitance across the silicon detectors is typically $C_{\mathrm{t}} \sim 10 \mathrm{pF}$. The load resistors used in this work are made of nickel-chromium thin film deposited on a

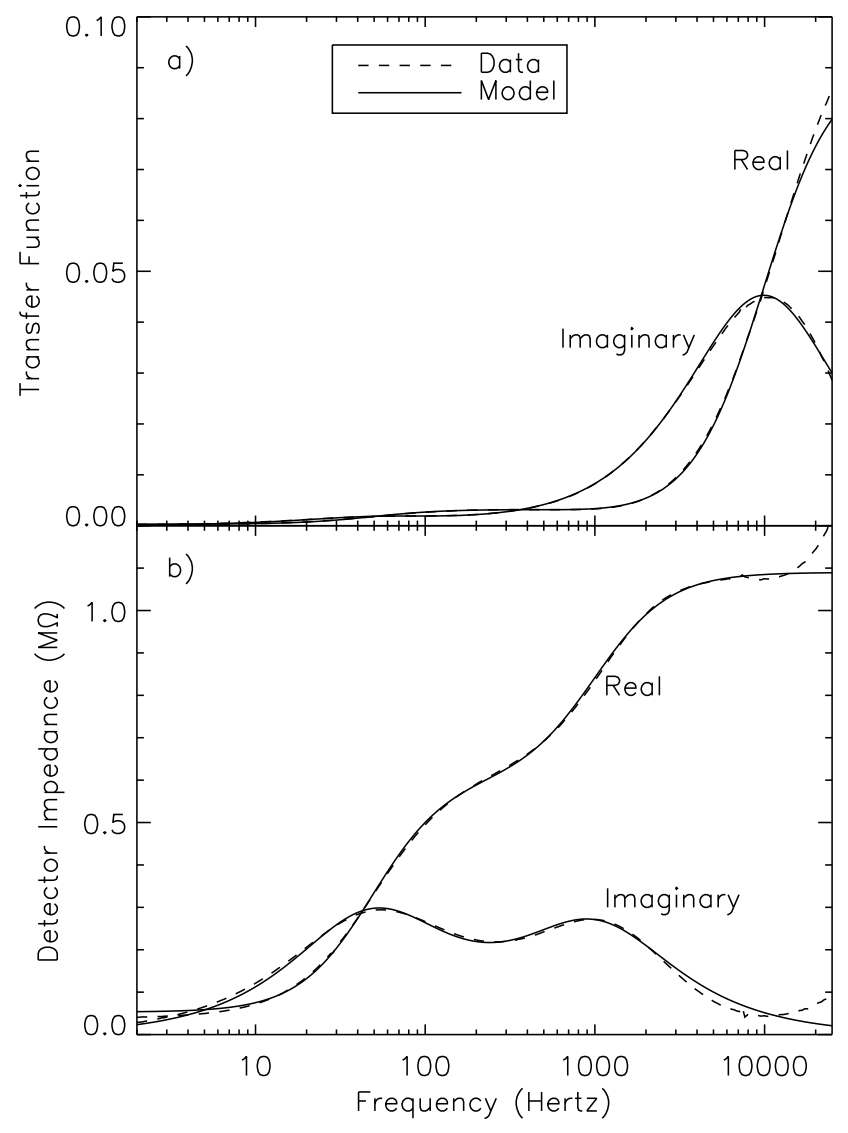

FIG. 5: Measured and fitted transfer functions of a biased detector with absorber. a) Transfer function. b) Detector impedance inferred from the transfer function using the measured stray capacitances.

thin silicon nitride insulating layer on a degenerate silicon substrate $^{20}$. These were found to have shunt capacitances on the order of $C_{\mathrm{L}} \sim 1 \mathrm{pF}$. A set of Nichrome resistors on quartz substrates ${ }^{20}$ had shunt capacitances an order of magnitude smaller.

These two shunt capacitances alone are not always sufficient to fit the unbiased transfer functions at high frequencies. In an effort to better model the distributed capacitance in the load resistor the resistance is split into two pieces and a third shunt capacitor is added, $C_{\mathrm{b}}$ [Fig. 3(a)]. Typical values of these additional components are $C_{b} \lesssim 1 \mathrm{pF}$ and $R_{L_{1}} / R_{L_{2}} \approx 1-5$.

Example fits of an unbiased transfer function are shown in Fig. 4. Including the effects of the stray capacitances in fitting the unbiased data should result in a completely real (resistive) impedance which is independent of frequency. The lower panel of Fig. 4 makes it clear that our simplest stray model $\left(C_{\mathrm{b}}=0\right)$ accomplishes this only at frequencies below $500 \mathrm{~Hz}$. If we include the distributed capacitance in the load resistor $\left(C_{\mathrm{b}} \neq 0\right)$ the effects can be removed up to frequencies of $\approx 1 \mathrm{kHz}$. The remaining deviations above $1 \mathrm{kHz}$ indicate that our stray models are not an adequate representation of the real 
circuit at these frequencies.

\section{Biased Transfer Functions}

In Fig. 5(a) we measure the transfer function of a biased detector (dotted lines). Using this transfer function and the measured values of the load resistor and stray capacitances we can calculate the impedance of the thermal detector from Eq. (23) or its equivalent; this impedance is shown as the dotted lines in Fig. 5(b). The best fit to the biased transfer function [solid line in Fig. 5(a)] is obtained by varying the detector parameters (i.e. $\alpha, \beta$, $C$ 's, $G$ 's) while the strays remain fixed at their measured values. The impedance for these best fit detector parameters is shown as the solid lines in Fig. 5(b). As already mentioned in Sec. III, the divergence at high frequencies is most likely due to inadequate modeling of the stray capacitances.

\section{Acknowledgments}

The doped silicon thermometers and their absorbers were fabricated by Regis Brekosky, Caroline Kilbourne, and colleagues at NASA/Goddard Space Flight Center. We thank Lindsay Rocks, Dahai Liu, and Melanie Clarke for their contributions to data analysis and acquisition. We would also like to thank Wilt Sanders, Enectali Figueroa-Feliciano, and Massimiliano Galeazzi for many useful discussions and especially Dan McCammon and Mark Lindeman for discussions and careful readings of this manuscript. This work has been supported by NASA grant NAG5-5404. a Current address: Enrico Fermi Institute, University of Chicago, 5640 S. Ellis Ave., Chicago, IL 60637; johnv@oddjob.uchicago.edu

1 R. C. Jones, J. Opt. Soc. Am. 43, 1 (1953).

2 J. C. Mather, Appl. Opt. 21, 1125 (1982).

3 J. C. Mather, Appl. Opt. 23, 3181 (1984), erratum: Appl. Opt. 24, 1407 (1985).

4 S. H. Moseley, J. C. Mather, and D. McCammon, J. Appl. Phys. 56, 1257 (1984).

5 S. F. Paul, R. J. Fonck, and A. K. MacAulay, Rev. Sci. Instrum. 64, 2423 (1993).

6 M. Galeazzi and D. McCammon, J. Appl. Phys. 93, 4856 (2003).

7 J. E. Vaillancourt, C. A. Allen, R. Brekosky, A. Dosaj, M. Galeazzi, R. Kelley, D. Liu, D. McCammon, F. S. Porter, L. E. Rocks, W. T. Sanders, and C. K. Stahle, Nucl. Instrum. Methods Phys. Res. A 520, 212 (2004).

8 M. Galeazzi, K. R. Boyce, G. V. Brown, C. Chen, J. Cottam, E. Figueroa-Feliciano, M. B. Jacobson, R. L. Kelley, D. Liu, D. McCammon, F. S. Porter, L. E. Rocks, C. K. Stahle, A. E. Szymkowiak, and J. E. Vaillancourt, Nucl. Instrum. Methods Phys. Res. A 520, 469 (2004).

9 J. C. Mather, Appl. Opt. 23, 584 (1984).

10 S. R. Bandler, E. Figueroa-Feliciano, C. K. Stahle, K. Boyce, R. Brekosky, J. Chervenak, F. Finkbeiner, R. Kelley, M. Lindeman, F. S. Porter, and T. Saab, Nucl. Instrum. Methods Phys. Res. A 520, 285 (2004).

${ }^{11}$ L. Giannone, K. Mast, and M. Schubert, Rev. Sci. Instrum. 73, 3205 (2002).
12 A. Murari, M. Cecconello, L. Marrelli, and K. F. Mast, Rev. Sci. Instrum. 75, 2692 (2004).

13 X. Gu, G. Karunasiri, G. Chen, U. Sridhar, and B. Xu, Appl. Phys. Lett. 72, 1881 (1998).

14 J. Schivell, G. Renda, J. Lowrance, and H. Hsuan, Rev. Sci. Instrum. 53, 1527 (1982).

15 M. A. Lindeman, S. Bandler, R. P. Brekosky, J. A. Chervenak, E. Figueroa-Feliciano, F. M. Finkbeiner, M. J. Li, and C. A. Kilbourne, Rev. Sci. Instrum. 75, 1283 (2004).

16 J. Zhang, W. Cui, M. Juda, D. McCammon, R. L. Kelley, S. H. Moseley, C. K. Stahle, and A. E. Szymkowiak, Phys. Rev. B 57, 4472 (1998).

17 D. McCammon, M. Galeazzi, D. Liu, W. T. Sanders, B. Smith, P. Tan, K. R. Boyce, R. Brekosky, J. D. Gygax, R. Kelley, D. B. Mott, F. S. Porter, C. K. Stahle, C. M. Stahle, and A. E. Szymkowiak, Phys. Status Solidi B 230, 197 (2002).

18 D. Liu, M. Galeazzi, D. McCammon, W. T. Sanders, B. Smith, P. Tan, K. R. Boyce, R. Brekosky, J. D. Gygax, R. Kelley, D. B. Mott, F. S. Porter, C. M. Stahle, C. K. Stahle, and A. E. Szymkowiak, AIP Conf. Proc. 605, 87 (2002).

19 T. Saab, E. Apodaca, S. R. Bandler, K. Boyce, J. Chervenak, E. Figueroa-Feliciano, F. Finkbeiner, C. Hammock, R. Kelley, M. Lindeman, F. S. Porter, and C. K. Stahle, Nucl. Instrum. Methods Phys. Res. A 520, 281 (2004).

20 purchased from Mini Systems, Inc., Attleboro, MA 02703 